\title{
Article \\ High-Order Resonant Peaks and Polarization Dependence of Microphotoluminescence in Whispering-Gallery Mode ZnO Microrod Cavity
}

\author{
Jiatian Guo ${ }^{\dagger}$, Huihui Wei ${ }^{\dagger}$, Jingyi Song, Yunhui Guo and Xiaobo Yuan * \\ School of Physics and Electronics, Shandong Normal University, Jinan 250038, China; \\ 2019020526@stu.sdnu.edu.cn (J.G.); 2018020535@stu.sdnu.edu.cn (H.W.); 201809020207@stu.sdnu.edu.cn (J.S.); \\ 2019020518@stu.sdnu.edu.cn (Y.G.) \\ * Correspondence: yxb@sdnu.edu.cn \\ + These authors contributed equally to this paper.
}

check for updates

Citation: Guo, J.; Wei, H.; Song, J.; Guo, Y.; Yuan, X. High-Order Resonant Peaks and Polarization Dependence of

Microphotoluminescence in Whispering-Gallery Mode $\mathrm{ZnO}$ Microrod Cavity. Crystals 2021, 11, 824. https://doi.org/10.3390/ cryst11070824

Academic Editor: Masakazu Anpo

Received: 31 May 2021

Accepted: 13 July 2021

Published: 15 July 2021

Publisher's Note: MDPI stays neutral with regard to jurisdictional claims in published maps and institutional affiliations.

Copyright: (c) 2021 by the authors. Licensee MDPI, Basel, Switzerland. This article is an open access article distributed under the terms and conditions of the Creative Commons Attribution (CC BY) license (https:// creativecommons.org/licenses/by/ $4.0 /)$.

\begin{abstract}
High order phenomena in the visible range and with polarization dependence in the ultraviolet (UV) region of the microphotoluminescence (micro-PL) spectrum in whispering-gallery mode (WGM) ZnO microrod cavity have been thoroughly studied at room temperature. WGM $\mathrm{ZnO}$ microrod cavity with good crystallinity is produced by the CVD growth method, and the $\mathrm{ZnO}$ microrod structures are characterized by structural and optical methods. Through the micro-PL spectrum measurement of the $\mathrm{ZnO}$ microrod, it is found that high-order resonance peaks appeared in the visible region. The different polarization conditions can be adjusted by rotating the angles of the polarizer, and it is proved that the micro-PL spectrum has strong polarization-dependent behavior in the UV region. Our results imply broad application potentials in the study of $\mathrm{ZnO}$ microrod-based photonic cavity devices.
\end{abstract}

Keywords: microrod cavity; whispering-gallery mode; high-order resonance phenomenon; polarization

\section{Introduction}

As an ideal semiconductor material, Zinc oxide $(\mathrm{ZnO})$ has a wide direct band gap and strong exciton binding energy, so the study of its optical properties has aroused great interest [1-4]. ZnO single crystal micro/nanostructure has shown great potential both in basic research and for practical applications in the fields of photoelectric and nanophotonic devices [5,6]. In addition, the micro/nanostructure of $\mathrm{ZnO}$ can also be used as a natural hexagonal whisper gallery mode (WGM) cavity. The WGM microcavity can achieve optical resonance through total internal reflection at the hexagonal boundary [7-9]. The $\mathrm{ZnO}$ WGM microcavity has become a research hotspot because it has a better laser mode, higher quality factor and lower laser threshold than other microcavities [7,10].

It is well known that microphotoluminescence (micro-PL) is a powerful imaging technique for studying exciton transition behavior. The room temperature micro-PL spectrum of the $\mathrm{ZnO}$ single crystal has been studied by many researchers from various aspects [4,11-14]. It is found that the typical $\mathrm{ZnO}$ luminescence is mainly concentrated on two bands: one is the UV region (shorter than $400 \mathrm{~nm}$ ), and the other is the visible region (mainly concentrated in region of 500-700 nm) [15,16]. Excellent luminescence properties appear both in the UV region and the visible region; therefore, $\mathrm{ZnO}$ has superior prospects for the research of UV or visible photon nanodevices $[17,18]$. Previous research on the optical properties of WGM microcavities mainly focused on changing the laser pump power density [19,20] or increasing the luminous intensity in the visible region [21] (so high quality factor (Q) and lower lasing threshold optical microcavities can be achieved), and the resonance peaks in the UV region induced by the Rabi splitting have also been studied [22,23]. However, there 
is still a lack of detailed investigations about the dependence of the resonance peaks on the polarization angle in the UV region.

The optical characteristics of a $\mathrm{ZnO}$ WGM microrod cavity grown on $\mathrm{SiO}_{2}$ substrate by the chemical vapor deposition method (CVD) at atmosphere pressure are investigated in this paper. It shows that the obtained $\mathrm{ZnO}$ microrod can act as a WGM resonator, in which WGM optical characterization is observed in the visible and UV range of the micro-PL spectrum. The WGM microcavity $\mathrm{ZnO}$ microrods exhibit high order characteristics in the visible region of the micro-PL spectrum. Due to the resonant coupling of excitons and WGM cavity modes, a new coupling system is formed in the UV region. By adjusting the polarization angle, it is found that the new composite coupling system has a strong dependence on the polarization. Our work reveals the high order phenomena and polarization dependence of micro-PL in WGM ZnO microrod cavities, which implies wide application potential in the study of $\mathrm{ZnO}$ microrod-based UV lasers, high-sensitivity detectors and optoelectronic devices.

\section{Materials and Methods}

$\mathrm{ZnO}$ microrods were grown on $\mathrm{SiO}_{2}$ substrates by the $\mathrm{CVD}$ method. $\mathrm{ZnO}$ microrods are prepared by $\mathrm{ZnO}$ powder and carbon powder at $1000{ }^{\circ} \mathrm{C}$ in Argon for $10 \mathrm{~min}$, and the flow rate of argon is controlled to $110 \mathrm{sccm}$. The $\mathrm{SiO}_{2}$ substrate is placed $51.5 \mathrm{~cm}$ away from the furnace mouth, the substrate is about $5 \mathrm{~cm}$ away from the source powder, and the temperature at the substrate is about $700{ }^{\circ} \mathrm{C}$ A carrier gas is necessary, because the vapor pressures of $\mathrm{O}$ and $\mathrm{Zn}$ are both lower than that of $\mathrm{ZnO}$ at these temperatures. Reaction boats are placed in a horizontal quartz furnace tube [24].

\section{Results and Discussion}

As shown in Figure 1a, the sample morphologies and the size of the hexagonal $\mathrm{ZnO}$ microrod were observed by using a scanning electron microscope (SEM, ZEISS Sigma 500, ZEISS, Germany). We found that the $\mathrm{ZnO}$ microrod had lengths of $\sim 10 \mu \mathrm{m}$ with a uniform diameter of $\sim 3 \mu \mathrm{m}$. The SEM image in Figure $1 \mathrm{~b}$ shows that the $\mathrm{ZnO}$ microrod had good crystallinity with a regular hexagonal cross section and a smooth surface. Thus, the total reflection effect occurred at the interface between the $\mathrm{ZnO}$ material and the air, which can confine the light in the WGM resonator. Previous studies reported that the energy difference between the cavity modes and the excitons can be controlled by modulating the radius of the $\mathrm{ZnO}$ microrod, so that the energy and the resonance order of the WGM mode can be tuned [25]. Figure $1 \mathrm{c}$ shows the light path diagram in the hexagonal cross section of $\mathrm{ZnO}$ microrod. A $\mathrm{ZnO}$ microrod was selected to measure its micro-PL spectrum by confocal micro-PL systems (UHTS 300 SMFC VIS, WITec, Germany, and JY-Horiba LabRam HR800 UV, HORIBA Jobin Yvon, France) equipped with a continuous wave He-Cd laser at $488 \mathrm{~nm}$ and $325 \mathrm{~nm}$ as excitation source. The diameter of the laser spot focused on the sample was less than submicron, and the scanning step along the $\mathrm{ZnO}$ microrod surface is measured from a microscale down to $\sim 4 \mu^{2}$, a focal length of 550 and a liquid nitrogen cooled CCD as the detector. The sample and objective could be moved independently in the $x-y$ and $x-y-z$ directions, respectively. All measurements experiments were recorded at room temperature.

Energy dispersive spectrometer (EDS) (Figure 2b-d) is an effective technique for the elemental analysis of materials. The energy spectrum of the prepared $\mathrm{ZnO}$ microrod structure was tested to analyze the main components of the $\mathrm{ZnO}$ microrod and the substrate. Through the test pattern, it was found that there were two elements present, $\mathrm{O}$ and $\mathrm{Zn}$, and they were evenly distributed on the surface of the $\mathrm{ZnO}$ microrods, which proves that the prepared $\mathrm{ZnO}$ microrods have very good expected composition without introducing other impurities. Every dot corresponds to a specific element (Zn, O) marked by a designated color which also indicates that the microstructure of the microrods is uniform. The elemental quantitative analysis of the EDS spectroscopy showed that the atom ratio of $\mathrm{O}$ and $\mathrm{Zn}$ is almost 1:1, as seen in Figure 2d, which further confirms that there are no dopants 
in the $\mathrm{ZnO}$ microrods. The results of the EDS and SEM experiments show that regular $\mathrm{ZnO}$ WGM microcavities without other impurities can be formed under the above experimental conditions.

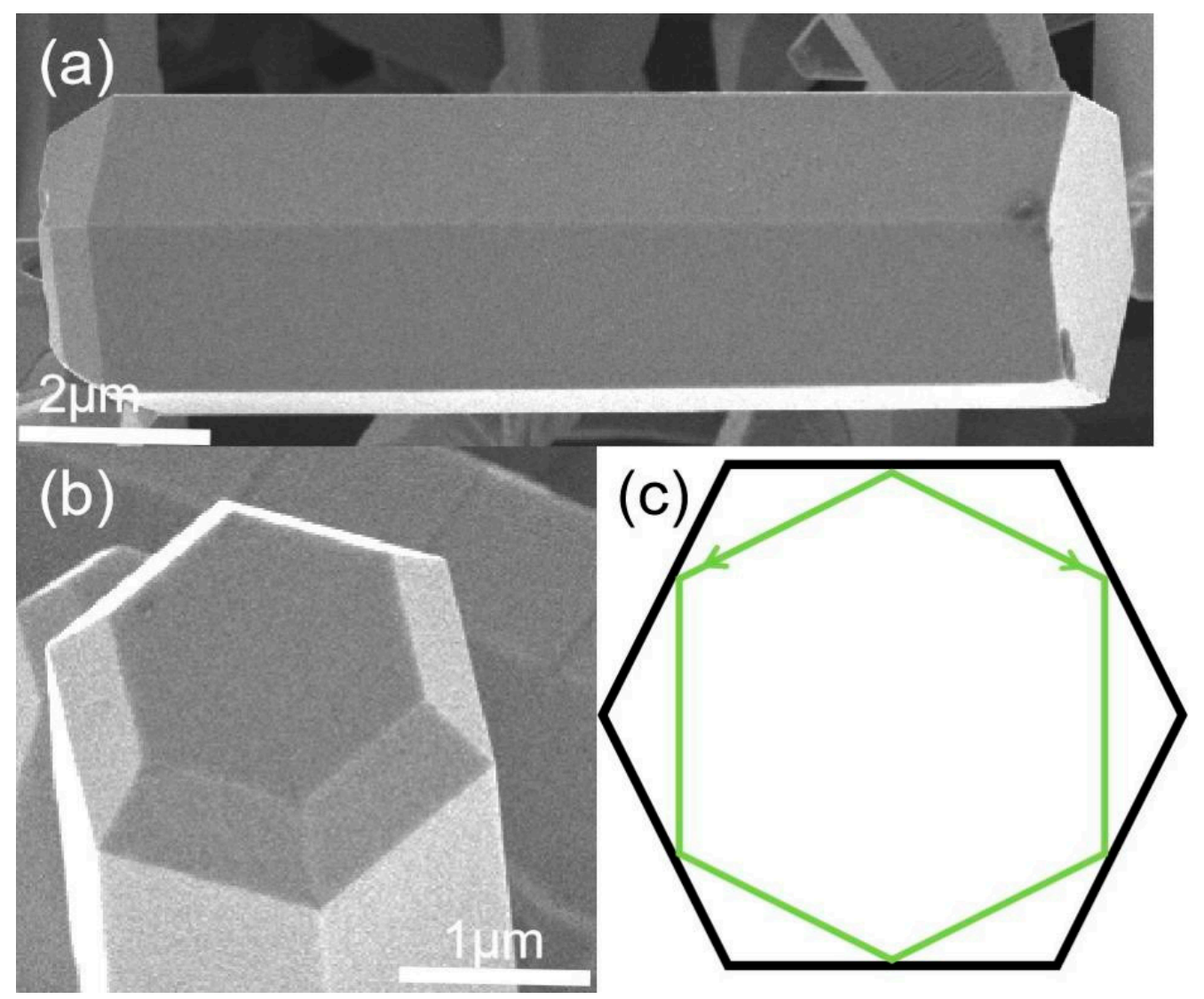

Figure 1. (a) SEM image of the prepared hexagonal $\mathrm{ZnO}$ microrod. (b) End face of $\mathrm{ZnO}$ microrod. (c) The schematic of hexagonal cross section of $\mathrm{ZnO}$ microrod supporting WGM resonances.

Visible region generation is ordinarily complicated owing to the multiple transition contributions and the existence of the multiple emissions [26-29]. In addition, the crystal morphology of $\mathrm{ZnO}$ microrods has a significant effect on the micro-PL spectrum [27,30-32]. In order to measure the properties of the resonant peaks in the visible region in detail, we used a $488 \mathrm{~nm}$ laser to perform a step-by-step micro-PL spectrum measurement on different positions of the $\mathrm{ZnO}$ microrod. Figure 3a shows the visible region micro-PL spectrum from $488 \mathrm{~nm}-525 \mathrm{~nm}$ at different positions of the horizontal ZnO WGM microcavity: it can be observed that there are continuous high-order peaks at $497 \mathrm{~nm}, 498 \mathrm{~nm}$ and 500 $\mathrm{nm}(\mathrm{A}, \mathrm{B}, \mathrm{C}$ in Figure 3a, respectively). The peak intensity is strongest when the measured point is in the middle. Since $\mathrm{ZnO}$ microrods can form a perfect WGM microcavity, it is necessary to discuss the effects of different orientations on the high-order resonant peaks in $\mathrm{ZnO}$ microrods. Figure $3 \mathrm{~b}$ shows the same visible region micro-PL spectrum at different positions of the vertical ZnO WGM microcavity; the high-order peaks are also observed at the different positions. Due to the symmetry, curve 1 and curve 3 are, in principle, the same. Here, the peak intensity of the curve 1 is weaker than that of the curve 3 -this is because that the laser spot position of curve 1 is at the extreme edge of $\mathrm{ZnO}$, so in this case only a small part of the excitation light enters the $\mathrm{ZnO}$ microrod. The resonance peak positions match with the theoretical mode very well $[33,34]$. At the same time, it can be shown that $\mathrm{A}, \mathrm{B}$ and $\mathrm{C}$ are the high order peaks generated by WGM resonance, rather than the pure defect peaks [8]. 

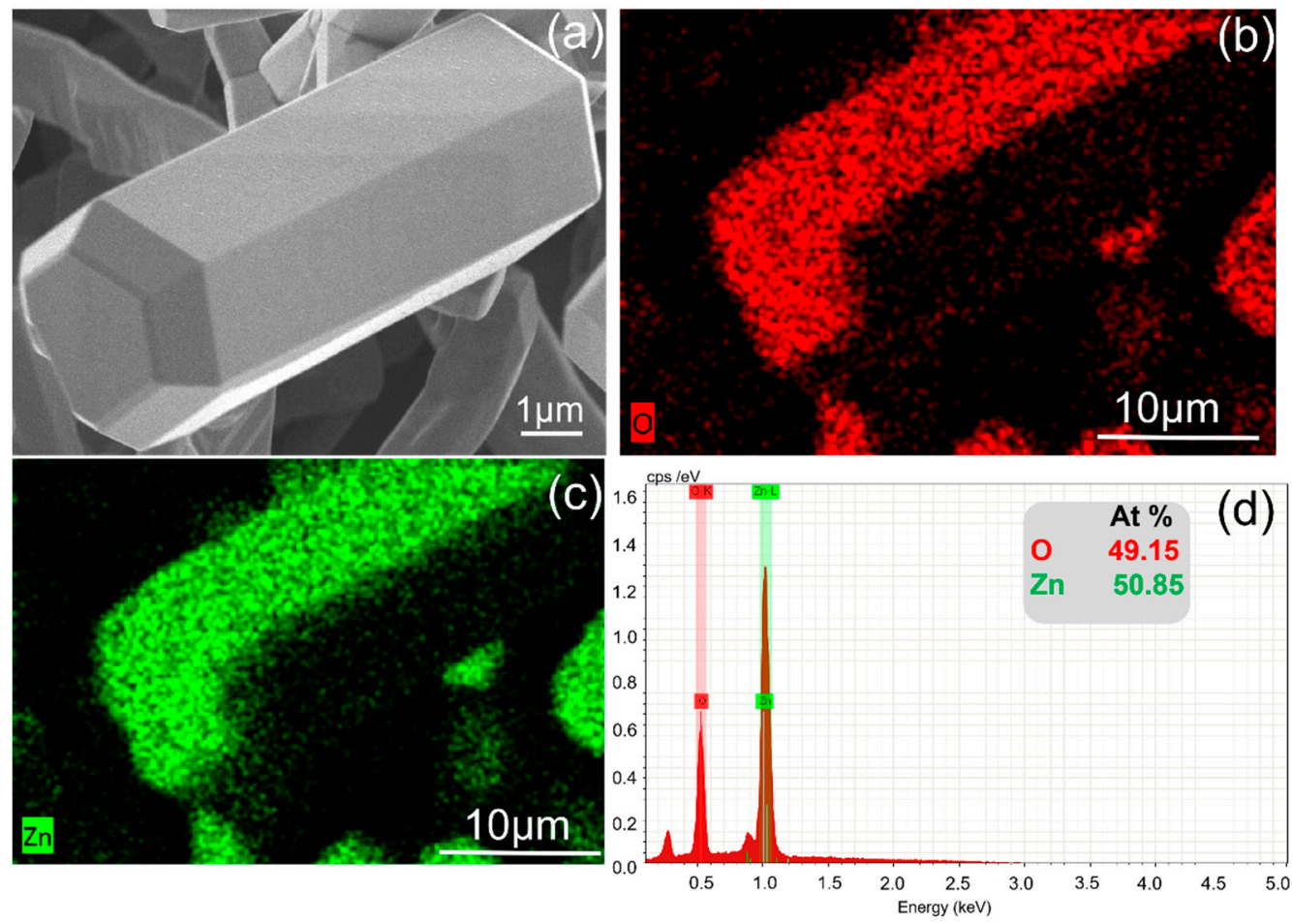

Figure 2. EDS spectroscopy-based elemental analysis of $\mathrm{ZnO}$ microrods. (a) SEM image of the selected hexagonal $\mathrm{ZnO}$ microrod. (b,c) EDS mapping. (d) EDS quantitative analysis.
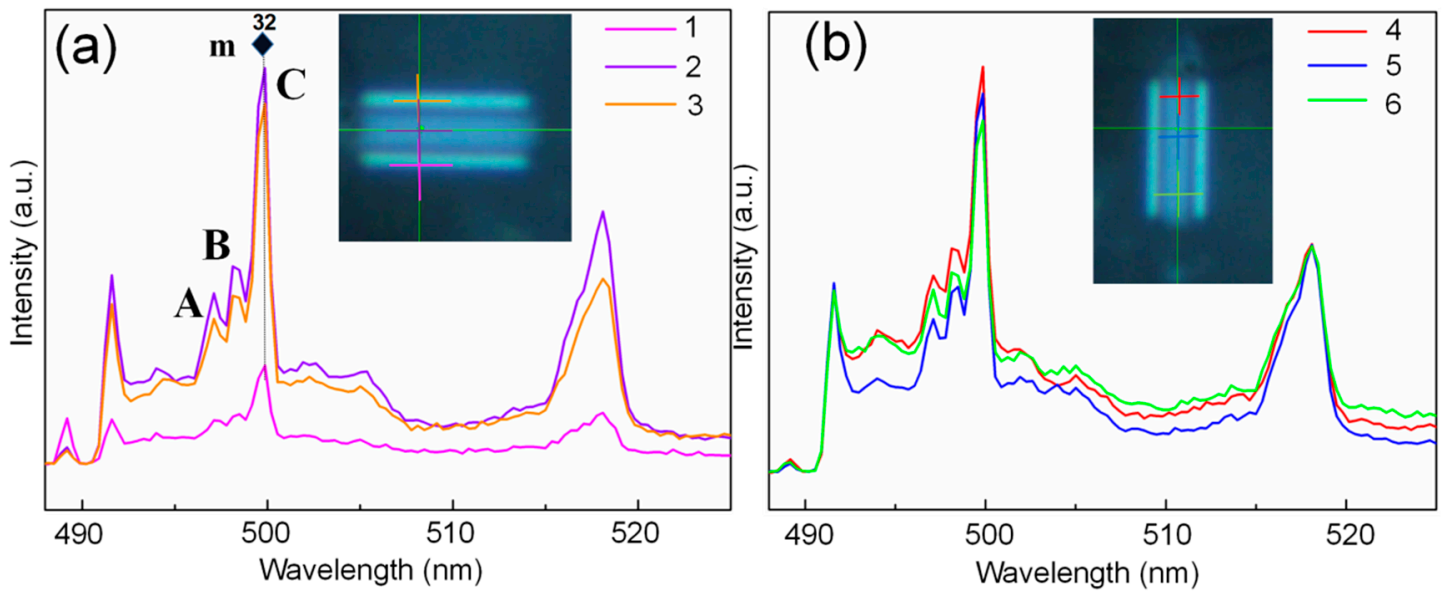

Figure 3. (a) The PL spectra at different positions of ZnO WGM microcavity were placed vertically, the WGM mode in the visible region is marked with the azimuthal mode number $\mathrm{m}$. Solid hexagon symbol represents the numerical simulation values; (b) PL spectra at different positions of ZnO WGM microcavity were placed horizontally.

The excitation light with a wavelength of $325 \mathrm{~nm}$ was used to obtain the micro-PL spectrum in the UV region at the incident light path, and two resonant peaks appeared at $378 \mathrm{~nm}$ and $388 \mathrm{~nm}$. In order to study the dependence of the properties of these two peaks on the polarization angle, the micro-PL spectrum was measured for each position of $\mathrm{ZnO}$ WGM with the polarization angles changes from $0^{\circ}-90^{\circ}$. When the crystal axis of the polarizer is parallel to the c-axis of the $\mathrm{ZnO}$ microrod, it is $0^{\circ}$. In order to the comprise the intensities of the two resonance peaks in the UV region with the polarization angles, five angles of $0^{\circ}, 23^{\circ}, 45^{\circ}, 68^{\circ}$ and $90^{\circ}$ were selected for analysis. The spatial positions also affect the WGM cavity mode, so three different positions were selected to compare and explore the polarization-dependent behavior of $\mathrm{ZnO} W G M$. The absolute 
intensity and the normalized relative intensity of the measured micro-PL spectrum were also compared. Figure $4 \mathrm{a}-\mathrm{c}$ show the optical images of the laser spots on the left, middle and right edges of the $\mathrm{ZnO}$ WGM. From Figure $4 \mathrm{~d}-\mathrm{f}$, it is found that the intensity of the two peaks is decreased with the increase in the angles for the three different positions, while the decrease in intensity for the peak at $378 \mathrm{~nm}$ is more pronounced. The microPL spectrum shows that the two WGM resonance peaks have a wide full width at half maximum (FWHM, as shown in Table 1). When the polarization angle is $0^{\circ}$, the FWHM of the peak at $388 \mathrm{~nm}$ is about $13.6 \mathrm{~nm}$, and it is about $7.4 \mathrm{~nm}$ for the peak at $378 \mathrm{~nm}$. With the increase in the polarization angle, blue shift of the two resonance peaks appears in the UV region, and the intensities of the two resonance peaks decrease; on the other hand, the FWHM of the peak increases at $388 \mathrm{~nm}$, while it decreases at $378 \mathrm{~nm}$.
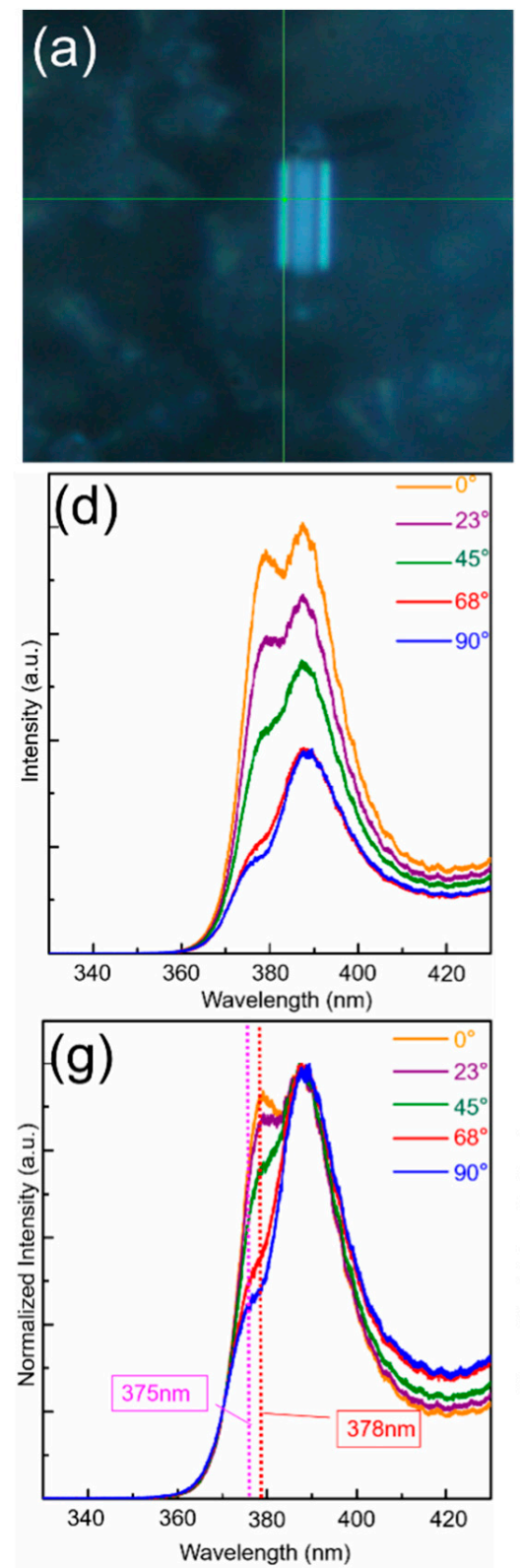
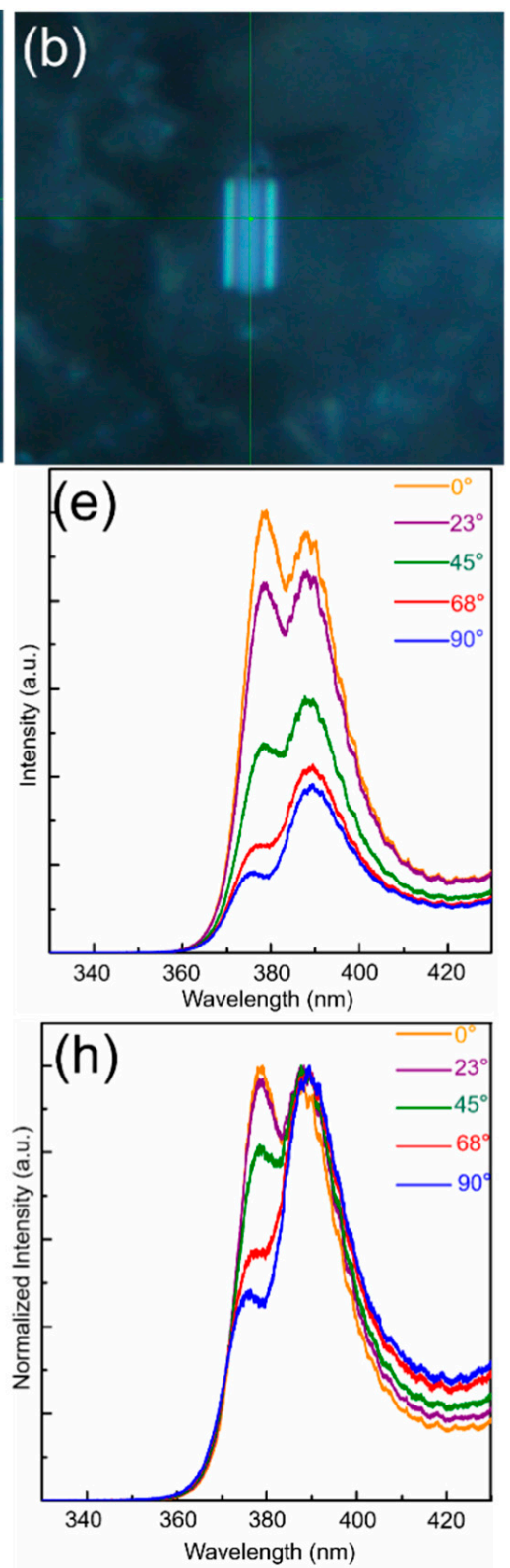
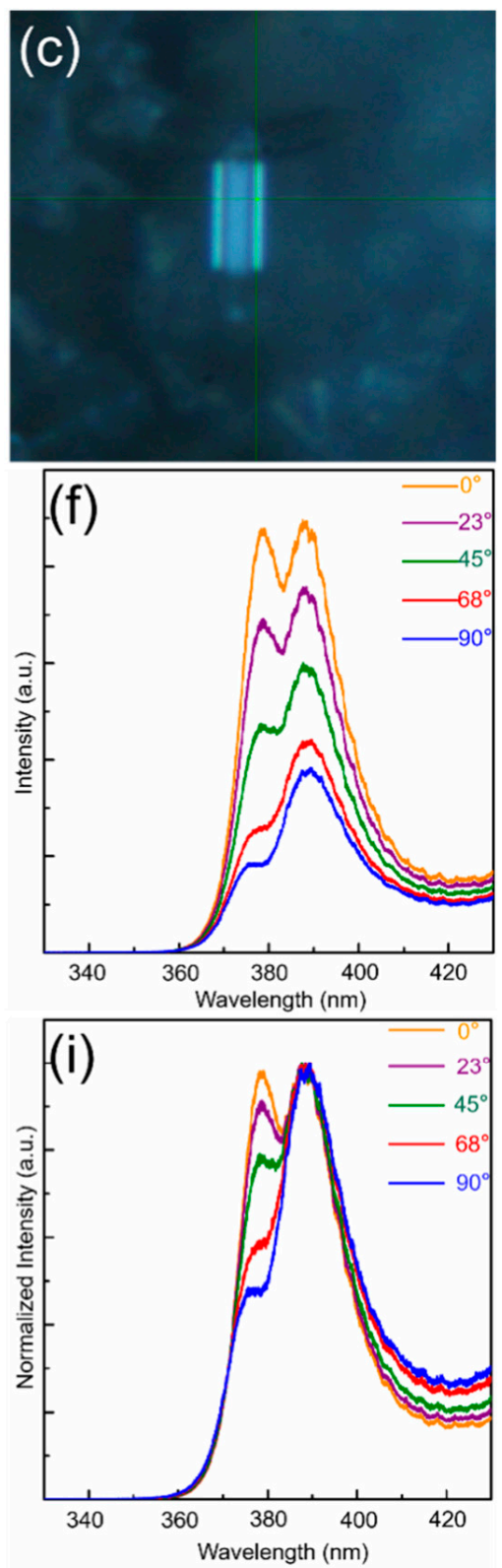

Figure 4. Polarization dependence of ZnO WGM on the different positions with different polarization angles. (a) Left edge position, (b) middle position, (c) right edge position. (d-f) and (g-i) are the polarization dependence of the ZnO WGM under the absolute intensity and the normalized intensity, respectively. For the PL spectrum in the UV region at each polarization angle, the maximum intensity is normalized to clearly show the polarization dependence. 
Table 1. The peak intensity and FWHM corresponding to the peak position at different polarization angles.

\begin{tabular}{cccc}
\hline Polarization Angle & Position (nm) & Intensity (a.u.) & FWHM \\
\hline $0^{\circ}$ & 378.2 & $13,153.6$ & 7.4 \\
$0^{\circ}$ & 388.5 & $16,630.5$ & 13.6 \\
$23^{\circ}$ & 378.1 & 9803.1 & 7.2 \\
$23^{\circ}$ & 388.4 & $13,977.2$ & 13.8 \\
$45^{\circ}$ & 377.8 & 6311.7 & 5.8 \\
$45^{\circ}$ & 388.3 & $11,744.2$ & 14.8 \\
$68^{\circ}$ & 377.1 & 1857.2 & 5.6 \\
$68^{\circ}$ & 388.2 & 8545.4 & 15.2 \\
$90^{\circ}$ & 375.5 & 1740.4 & 5.2 \\
$90^{\circ}$ & 388.1 & 7744.5 & 15.6 \\
\hline
\end{tabular}

After normalization, it was found that the intensity of the peak at $378 \mathrm{~nm}$ decreases as the polarization angle increases, and the phenomenon of blue shift occurs, as shown in Figure $4 \mathrm{~g}-\mathrm{i}$. These results indicate that the intensity changes of the two peaks have a strong dependence on the polarization, and the peak at the $378 \mathrm{~nm}$ has the greatest dependence. As mentioned in previous reports, the generation of resonance peaks in the UV region is mainly due to the selective transition rule of A exciton, B exciton and C exciton; A exciton has polarization properties, so the fluorescence intensity of TE polarization is higher than that of TM polarization, and the transition selectivity rule determines that A exciton is a complete TE polarization, B exciton has a certain transition probability under TE and TM polarization, while $C$ exciton is mainly TM polarization $[18,19,25,32]$. According to the experimental results, the $0^{\circ}$ polarization angle corresponds to the TE polarized light: in this case the light collected by the WGM microcavity is the light of the A and B exciton transitions, which exhibits strong intensity. When angles change, the collected light is mainly the light of the C exciton transition, i.e., mainly TM polarized light is collected, and all TE polarized light is filtered out, so the two peaks intensity are weak. Especially, the relative intensity of the peak at $378 \mathrm{~nm}$ attenuates more significantly. On the other hand, the micro-PL peak blueshift from $378 \mathrm{~nm}$ to $375 \mathrm{~nm}$ can be found by comparing the micro-PL spectra in Figure 4d, and the blueshift is gradually formed owing to the exciton polarization. As the polarization angle increases, the light that mainly collects the A and B exciton transitions is changed to the light that mainly transitions from the C exciton [25,35]. Given the above, the $\mathrm{C}$ exciton corresponds to the shortest transition wavelength and the highest energy. Therefore, a gradual blue shift from the low energy side to the high energy side appears in the $\mathrm{ZnO}$ WGM.

\section{Conclusions}

In summary, we reported the high order phenomenon in the visible range and the polarization-dependent behavior in the UV region of the micro-PL spectrum in ZnO WGM, which was prepared by CVD method. The obtained results demonstrate the micro-PL has strong dependence on the polarization in the UV region. In the UV region, the polarization mainly affects the transition effects of the excitons, so the distribution of the polarized light modes can be observed. However, the polarization mainly affects the excitation intensity in the UV region. With the increase in the polarization angle, the intensities of the two resonance peaks in the UV region are weakened, which is caused by the selective transition of excitons. At the same time, we proved that the TE polarization mode is dominant in the two resonance peaks in the UV region. Additionally, with the increase in the polarization angle, blue shift appears for the resonant peak at $378 \mathrm{~nm}$. The results show that the WGM $\mathrm{ZnO}$ microrod cavity may provide favorable conditions for the progress of novel polarized devices. 
Author Contributions: Data curation, Formal analysis, Visualization, Writing—review and editing, J.G.; Data curation, Formal analysis, Investigation, Methodology, Visualization, Writing-original draft, H.W.; Resources, Software J.S., Y.G.; Project administration, Resources, Supervision, Funding acquisition, Writing-review and editing, X.Y. All authors have read and agreed to the published version of the manuscript.

Funding: The authors would like to acknowledge the financial support from the Natural Science Foundation of Shandong Province (Grant No. ZR2018MA042).

Conflicts of Interest: The authors declare no conflict of interest.

\section{References}

1. Ton-That, C.; Weston, L.; Phillips, M. Characteristics of point defects in the green luminescence from Zn- and O-rich ZnO. Phys. Rev. B 2012, 86, 115205. [CrossRef]

2. Ton-That, C.; Zhu, L.; Lockrey, M.; Phillips, M.; Cowie, B.C.C.; Tadich, A.; Thomsen, L.; Khachadorian, S.; Schlichting, S.; Jankowski, N.; et al. Molecular nitrogen acceptors in ZnO nanowires induced by nitrogen plasma annealing. Phys. Rev. B 2015, 92, 024103. [CrossRef]

3. Shokri, A.; Yazdani, A.; Rahimi, K. Tunable electronic and optical properties of g-ZnO/ $\alpha-\mathrm{PtO}_{2}$ van der Waals hetero-structure: A density functional theory study. Mater. Chem. Phys. 2020, 255, 123617. [CrossRef]

4. Zhang, X.; Qin, J.; Xue, Y.; Yu, P.; Zhang, B.; Wang, L.; Liu, R. Effect of aspect ratio and surface defects on the photocatalytic activity of $\mathrm{ZnO}$ nanorods. Sci. Rep. 2015, 4, 4596. [CrossRef]

5. Van Vugt, L.K.; Rühle, S.; Ravindran, P.; Gerritsen, H.C.; Kuipers, L.; Vanmaekelbergh, D. Exciton Polaritons Confined in a ZnO Nanowire Cavity. Phys. Rev. Lett. 2006, 97, 147401. [CrossRef] [PubMed]

6. Sturm, C.; Hilmer, H.; Rheinländer, B.; Schmidt-Grund, R.; Grundmann, M. Cavity-photon dispersion in one-dimensional confined microresonators with an optically anisotropic cavity material. Phys. Rev. B 2011, 83, 205301. [CrossRef]

7. Dai, J.; Xu, C.X.; Zheng, K.; Lv, C.G.; Cui, Y.P. Whispering gallery-mode lasing in ZnO microrods at room temperature. Appl. Phys. Lett. 2009, 95, 241110. [CrossRef]

8. Xu, C.; Dai, J.; Zhu, G.; Zhu, G.; Lin, Y.; Li, J.; Shi, Z. Whispering-gallery mode lasing in ZnO microcavities. Laser Photon. Rev. 2014, 8, 469-494. [CrossRef]

9. Chen, R.; Ling, B.; Sun, X.W.; Sun, H.D. Room Temperature Excitonic Whispering Gallery Mode Lasing from High-Quality Hexagonal ZnO Microdisks. Adv. Mater. 2011, 23, 2199-2204. [CrossRef]

10. Xu, C.; Qin, F.; Zhu, Q.; Lu, J.; Wang, Y.; Li, J.; Lin, Y.; Cui, Q.; Shi, Z.; Manohari, A.G. Plasmon-enhanced ZnO whisper-ing-gallery mode lasing. Nano Res. 2018, 11, 3050-3064. [CrossRef]

11. Teke, A.; Özgür, Ü.; Doğan, S.; Gu, X.; Morkoç, H.; Nemeth, B.; Nause, J.; Everitt, H.O. Excitonic fine structure and recom-bination dynamics in single-crystalline ZnO. Phys. Rev. B 2004, 70, 195207. [CrossRef]

12. Djurisic, A.B.; Leung, Y.H. Optical properties of ZnO nanostructures. Small 2006, 2, 944-961. [CrossRef]

13. Damberga, D.; Fedorenko, V.; Grundšteins, K.; Altundal, Ş.; Šutka, A.; Ramanavičius, A.; Coy, E.; Mrówczyński, R.; Iatsunskyi, I.; Viter, R. Influence of PDA Coating on the Structural, Optical and Surface Properties of ZnO Nanostructures. Nanomaterials 2020, 10, 2438. [CrossRef] [PubMed]

14. Kominis, I.K.; Kornack, T.W.; Allred, J.C.; Romalis, M.V. A subfemtotesla multichannel atomic magnetometer. Nat. Cell Biol. 2003, 422, 596-599. [CrossRef] [PubMed]

15. Rodnyi, P.A.; Khodyuk, I.V. Optical and luminescence properties of zinc oxide (Review). Opt. Spectrosc. 2011, 111, 776-785. [CrossRef]

16. Ramanachalam, M.S.; Rohatgi, A.; Carter, W.B.; Schaffer, J.P.; Gupta, T.K. Photoluminescence study of ZnO varistor stability. J. Electron. Mater. 1995, 24, 413-419. [CrossRef]

17. Divins, N.J.; Kordus, D.; Timoshenko, J.; Sinev, I.; Zegkinoglou, I.; Bergmann, A.; Chee, S.W.; Widrinna, S.; Karslığlu, O.; Mistry, $\mathrm{H}$; i et al. Operando high-pressure investigation of size-controlled $\mathrm{CuZn}$ catalysts for the methanol synthesis reaction. Nat. Commun. 2021, 12, 1-10. [CrossRef]

18. Volkov, V.V.; Oliver, D.J.; Perry, C.C. Polariton condensation and surface enhanced Raman in spherical ZnO micro-crystals. Nat. Commun. 2020, 11, 4908. [CrossRef]

19. Dai, J.; Xu, C.X.; Ding, R.; Zheng, K.; Shi, Z.L.; Lv, C.G.; Cui, Y.P. Combined whispering gallery mode laser from hexag-onal ZnO microcavities. Appl. Phys. Lett. 2009, 95, 191117. [CrossRef]

20. Flatae, A.M.; Burresi, M.; Zeng, H.; Nocentini, S.; Wiegele, S.; Parmeggiani, C.; Kalt, H.; Wiersma, D. Optically controlled elastic microcavities. Light Sci. Appl. 2015, 4, e282. [CrossRef]

21. Evlyukhin, A.B.; Novikov, S.M.; Zywietz, U.; Eriksen, R.L.; Reinhardt, C.; Bozhevolnyi, S.I.; Chichkov, B.N. Demonstra-tion of magnetic dipole resonances of dielectric nanospheres in the visible region. Nano Lett. 2012, 12, 3749-3755. [CrossRef]

22. Gargas, D.J.; Moore, M.C.; Ni, A.; Chang, S.-W.; Zhang, Z.; Chuang, S.-L.; Yang, P. Whispering Gallery Mode Lasing from Zinc Oxide Hexagonal Nanodisks. ACS Nano 2010, 4, 3270-3276. [CrossRef] [PubMed] 
23. Willander, M.; Nur, O.; Zhao, Q.; Yang, L.; Lorenz, M.; Cao, B.; Pérez, J.Z.; Czekalla, C.; Zimmermann, G.; Grundmann, M. Zinc oxide nanorod based photonic devices: Recent progress in growth, light emitting diodes and lasers. Nanotechnology 2009, 20, 332001. [CrossRef] [PubMed]

24. Nowak, E.; Szybowicz, M.; Stachowiak, A.; Piechowiak, D.; Miklaszewski, A.; Witkowski, M.; Makowski, M.; Drozdowski, W.; Paprocki, K.; Fabisiak, K.; et al. The Influence of Recrystallization on Zinc Oxide Microstructures Synthesized with Sol-Gel Method on Scintillating Properties. Crystals 2021, 11, 533. [CrossRef]

25. Sun, L.; Chen, Z.; Ren, Q.; Yu, K.; Bai, L.; Zhou, W.; Xiong, H.; Zhu, Z.Q.; Shen, X. Direct Observation of Whispering Gallery Mode Polaritons and their Dispersion in a ZnO Tapered Microcavity. Phys. Rev. Lett. 2008, 100, 156403. [CrossRef]

26. Tian, Z.R.; Voigt, J.A.; Liu, J.; McKenzie, B.; McDermott, M.J.; Rodriguez, M.A.; Konishi, H.; Xu, H. Complex and oriented ZnO nanostructures. Nat. Mater. 2003, 2, 821-826. [CrossRef]

27. Güell, F.; Martínez-Alanis, P.R. Tailoring the Green, Yellow and Red defect emission bands in ZnO nanowires via the growth parameters. J. Lumin. 2019, 210, 128-134. [CrossRef]

28. Shi, S.; Wang, P.; Cui, J.; Sun, Z. Microstructure and Doping/Temperature-Dependent Photoluminescence of ZnO Nano-spears Array Prepared by Hydrothermal Method. Nanoscale Res. Lett. 2018, 13, 223. [CrossRef]

29. Schlather, A.E.; Large, N.; Urban, A.; Nordlander, P.; Halas, N. Near-Field Mediated Plexcitonic Coupling and Giant Rabi Splitting in Individual Metallic Dimers. Nano Lett. 2013, 13, 3281-3286. [CrossRef]

30. Chen, J.; Zhang, J.; Dai, J.; Wu, F.; Wang, S.; Long, H.; Liang, R.; Xu, J.; Chen, C.; Tang, Z. Strain dependent anisotropy in photo-luminescence of heteroepitaxial nonpolar a-plane ZnO layers. Opt. Mater. Express 2017, 7, 3944-3951. [CrossRef]

31. Kołodziejczak-Radzimska, A.; Jesionowski, T. Zinc oxide-from synthesis to application: A review. Materials 2014, 7, $2833-2881$. [CrossRef] [PubMed]

32. Yanagida, T.; Fujimoto, Y.; Yamanoi, K.; Kano, M.; Wakamiya, A.; Kurosawa, S.; Sarukura, N. Optical and scintillation proper-ties of bulk ZnO crystal. Phys. Status Solidi C 2012, 9, 2284-2287. [CrossRef]

33. Yang, Y.-D.; Tang, M.; Wang, F.-L.; Xiao, Z.-X.; Xiao, J.-L.; Huang, Y.-Z. Whispering-gallery mode hexagonal mi-cro-/nanocavity lasers. Photonics Res. 2019, 7, 594-607. [CrossRef]

34. Yang, Y.-D.; Huang, Y.-Z. Symmetry analysis and numerical simulation of mode characteristics for equilateral-polygonal optical microresonators. Phys. Rev. A 2007, 76, 023822. [CrossRef]

35. Dai, J.; Xu, C.X.; Sun, X.W.; Zhang, X.H. Exciton-polariton microphotoluminescence and lasing from ZnO whispering-gallery mode microcavities. Appl. Phys. Lett. 2011, 98, 161110. [CrossRef] 